\title{
On The Theory Of Perfect Numbers and Primes
}

\author{
Alex Nguhi
}

\section{Abstract}

This paper explores the properties of the set $\frac{n(n+1)}{2}$ and its implication on the distribution of perfect numbers. A major takeaway is a conjecture that all perfect numbers - even and odd lie on that line. It also describes primes arising from the perfect number line equation and equivalent statements of perfectness.

Notation:

$n$ ! is n factorial,$n !=n \times(n-1) \times(n-2) \times(n-3) \times \ldots \times 1$

$n !^{+}$is the sum factorial, $n !^{+}=n+(n-1)+(n-2)+(n-3)+\ldots+1$

$\gamma \in \aleph$ is all possible integer values of the form $n !^{+}$

$\delta(n)=d_{i}$ is a divisor of $n$

\section{Introduction}

The theory of perfect numbers is one of the oldest problems in Number Theory. The problem has expanded our understanding of primes but much of it is still unknown, [1].

Some important questions arise, are there any even perfect numbers other than those of EuclidEuler Theorem ? [2] Are there any odd perfect numbers ? [3]

\section{Statement Of Results}

\subsection{The Sums $n !^{+}$}

The sum of all integers in the interval $[n, 1]$ can be defined by the equation $\frac{n(n+1)}{2}$

$$
\begin{aligned}
& 1=\frac{1 \times 2}{2}=1 \\
& 1+2=\frac{2 \times 3}{2}=3 \\
& 1+2+3=\frac{3 \times 4}{2}=6 \\
& 1+2+3+4=\frac{4 \times 5}{2}=10
\end{aligned}
$$




\subsection{Perfect Numbers}

A number $\gamma$ is perfect if and only if $\gamma=\sum_{i} d_{i}$ that is, a number is perfect if it is equal to the sum of its divisors(including 1 ).

\subsubsection{Even Perfect Numbers}

Proposition 1.1 If an even integer $\gamma$ is perfect then $\gamma \in \aleph$

\section{Proof}

This can be proven by showing that $\gamma$ is equivalent to the Euler-Euclid definition of perfect numbers which is:

$\gamma=2^{y-1}\left(2^{y}-1\right), y \in \mathbf{N}$ where $2^{y}-1$ is what is called a Mersenne prime, [4].

If so then $\gamma=\frac{n(n+1)}{2}$ then we can let $n$ be a Mersenne prime and without any loss of generality then we can say that $n+1$ is a power of the form $2^{y}$ that eventually leads to the value of $\gamma$ itself. This proposition is trivial but not obvious enough. A consequence of this is that we can make many deductions, hypotheses and conjectures regarding perfect numbers.

We'll begin with prime numbers then make calculated inferences on perfect numbers.

Proposition 1.2 For any integer $k, k=n !^{+}$, then $n$ and $n+1$ are co-primes

This solution again is trivial in that it can be proven from the Fundamental Theorem of Prime Numbers and Euclid's Postulate of infinite primes, [6,7].

From the proposition-

Theorem 1.1 For a number $k=n !^{+}$, there are infinitely many primes of the form $p=k \pm \delta(k) \pm 1$

When $d_{i}=1$ or 2

By definition $\gamma$ cannot be a prime number, therefore for very short intervals say for some conditional statements :-

1. If $k$ is odd then $p=k \pm 2$ then $p$ is relatively prime. If it's not prime we can employ fast calculating algorithms to prove it's not.

2. If $\mathrm{k}$ is even then we can have a modification such that $d=2$ so the prime number is the form $p=k+2-1$ such an equation is $p=k \pm 1$

This infers that a prime number exist in the region $k \pm s ; s=1,2,3$

Examples :

In the Ranges

$k=p_{i}$

$6=3,5,7$ 
$10=7,11,13$

$15=13,17$

$21=19,23$

$28=29,31$

$36=37$

$45=43,47$

Computer Algorithms involving modular arithmetic produce very high densities of prime numbers with high precision.

\section{Quadratic equations}

Suppose that $p=k+1+1$

Then:-

$$
\begin{gathered}
p=k+1+1 \\
p=\frac{n(n+1)}{2}+2 \\
p=\frac{n^{2}+n+4}{2}
\end{gathered}
$$

There are infinitely many primes of this with one characteristic - they have $\mathbf{3}$ and $\mathbf{7}$ as their only terminal digits except for 2 and 5

Observe $p=2,3,5,17,23,47,107,173,233, \ldots$

A generalization of primes of that form is:

$$
p=\frac{n^{2}+n+2^{y}}{2}
$$

When $d_{i}>2$

Consider $p=k \pm d_{i} \pm 1$

for $p=55 \pm 5 \pm 1=59,61$ primes.

These too exist because of the coprime nature of $n$ and $n+1$. This can then be extended for other coprimes. 
Primes from Coprimes of the form $\alpha n(n+1) \pm 1$

Some solutions for $p$ when $\alpha=2$ are are as follows

$p=3,11,23,59,83,179,263,311,419,479,683,839,1103,1511,2111,2243,2663,2963,3119,4139,4703,5099,5303$

$, 12959,17483,19403,21011,21839,22259,24419,25763,27143,27611,28559,30011,32003,33023,34583,35111$

,64439,67343,68819,75659,78803,81203,83639,86111,91163,95483,98123,100799,103511,107183,111863,1

There are many primes of this form and we'll examine why.

$$
\begin{gathered}
p=\left(n^{2}-1\right)+(n+1)^{2}-1 \\
n^{2}-1+n^{2}+2 n+1-1 \\
2 n^{2}+2 n-1 \\
2 n(n+1)-1
\end{gathered}
$$

The two integers $2 n$ and $n+1$ are coprimes and a -1 operation produces a relatively prime number.

More generally :-

$$
2 n(n+1) \pm 1
$$

When $\alpha=3$ so that $3 n(n+1) \pm 1$

For $3 n(n+1)-1$

$p=5419$

21419, 18959, 17099, 16649, 1699, 15767, 14489, 12479, 11717, 10979, 10239, 9239, 7649, 7349, 5939, 5417,

21929, 24029, 25667, 28517,

\subsubsection{Odd Perfect Numbers}

In the current mathematical atmosphere, odd perfect numbers haven't been defined, only certain conditions of how they might be like. Nonetheless, this leaves opportunity for hypotheses as stated earlier.

This can also be attributed to the fact that there's no coherent theorem describing the structure of prime numbers enough to get an irrefutable proposition.

Conjecture 1.1 If an odd perfect number $\gamma_{\theta}$ exists, then $\gamma_{\theta} \in \aleph$

This can be generalized into a Perfect Number Conjecture for both odd and even numbers : 


\subsubsection{Properties of Odd Perfect Numbers :}

Theorem 1.2 If $\gamma$ is perfect then none of the $n$ or $(n+1)$ can be prime unlike the even number solutions.

This can be generalized into an inequality if either is prime but not both( special case 2,3 )

$$
\frac{\gamma}{n} \leq \frac{n+1}{2}
$$

This is woven to the geometric property because only a power series produces the highest number divisors for the largest possible summation whilst not affecting the structure of $\gamma$ since we're only interested in the LCM of the $d_{i}$ which makes up the integer under evaluation.

For clarifications $\gamma=\sum_{i} d_{i}$ is not strictly perfect but just a sum involving $n$ and $n+1$

Theorem 1.3 For near perfect numbers, with the choice that neither $n$ and $n+1$ are not primes and have a high composition of powers

then:

$$
\frac{\gamma}{n} \geq \frac{n+1}{2}
$$

\subsubsection{Equivalent statements to the perfect number line.}

Statement 1: $\quad \gamma=2\left(\frac{n}{2}\right)^{2}-\frac{n}{2}$

if $\frac{n}{2}=w$

then

$$
\gamma=2 w^{2}-w
$$

Statement $2: \gamma \approx n^{3}-n^{2}-n$ for $n>1$

To be precise :

$$
\gamma=n^{3}-n^{2}-n+\epsilon
$$

for $n>1$ and $\epsilon$ having \pm flexibility. 


\section{Open Problems}

1. Does the series of successive pairs of odd numbers have a convergence point $c$ such as the one of even perfect numbers.

$$
c=\frac{n}{\frac{n+1}{2}}
$$

$c=2$ as $n \rightarrow \infty$ for even numbers.

2. Because there exists a weak correspondence between the set $\gamma \in \aleph$ and square numbers, then we can use this to expound on one of Landau's problems basing on our previous findings.

$$
p=a^{2}+1
$$

The weak correspondence tells us that there's a high probability of $p$ being prime.

Landau's hypothesis can be extended in such a way that if $a$ is prime, are there infinitely many primes of the form?

$$
p=a^{y}+1
$$

Again, the weak correspondence doesn't end with square numbers but extends to power integers .

For square numbers its value is like a moving average between subsequent values of $k$ defined earlier, observe

Consider an integer $x$

$$
\begin{gathered}
x=\frac{\frac{n(n+1)}{2}+\frac{(n+1)(n+2)}{2}}{2} \\
=\frac{n^{2}+2 n+1}{2}
\end{gathered}
$$

Hence :

$$
a^{y} \approx \frac{n^{2}+2 n+1}{2}
$$

Making :

$$
p \approx a^{y}+\epsilon
$$

Examples

$k=1,3,6,10,21,28,36,45,55,66$

$x=2,4.5,8,15.5,24,32,41,50$, 
3. The use of the geometric property of $\gamma=n^{3}-n^{2}-n+\epsilon$ to examine divisibility, primes and perfect integers.For instance inserting $w$ described previously but $w \in \mathbb{Z}$

$$
\gamma=w^{3}-w^{2}-w+\epsilon
$$

4. The relationship between $n$ ! and the equivalent equations.

$$
n ! \approx w^{3}-w^{2}-w
$$

\section{Conclusion}

There's a correlation between perfect numbers and the sum factorials of integers - even and odd (if they indeed do exist). 


\section{References}

[1]. Hare, K. (2005). More on the total number of prime factors of an odd perfect number. Mathematics of computation, 74(250), 1003-1008.

[2]. Everest, G., Stevens, S., Tamsett, D., \& Ward, T. (2007). Primes generated by recurrence sequences. The American Mathematical Monthly, 114(5), 417-431.

[3]. Hare, K. (2005). More on the total number of prime factors of an odd perfect number. Mathematics of computation, 74(250), 1003-1008.

[4]. Hoque, A., \& Saikia, H. K. (2014). On generalized Mersenne prime. SeMA Journal, 66(1), 1-7.

[5]. Pintz, J. (2009). Landau's problems on primes. Journal de théorie des nombres de Bordeaux, 21(2), 357-404.

[6]. Selberg, A. (1949). An elementary proof of the prime-number theorem. Annals of Mathematics, 305-313.

[7]. Meštrović, R. (2012). Euclid's theorem on the infinitude of primes: a historical survey of its proofs (300 BC-2017) and another new proof. arXiv preprint arXiv:1202.3670. 